\title{
Measuring the Noise Impedances of Switched-Mode Power Supplies
}

\author{
Enrico Mazzola \\ Schaffner Group, Automotive Division \\ and \\ Politecnico di Milano, DEIB \\ enrico.mazzola@schaffner.com
}

\author{
Flavia Grassi \\ Politecnico di Milano \\ $D E I B$ \\ 20133 Milan, Italy \\ flavia.grassi@polimi.it
}

\author{
Alessandro Amaducci \\ Schaffner Group \\ Automotive Division \\ 4542 Luterbach, Switzerland \\ alessandro.amaducci@ schaffner.com
}

\begin{abstract}
In this paper, a novel approach to complex impedance measurement is proposed, using an Impedance Analyzer (IA) connected to the system under investigation through a transformer, which allows direct measurement of the magnitude and phase of the common mode (CM) and differential mode (DM) impedance of a Switched-Mode Power Supply (SMPS) during working conditions. The theoretical analysis of the measurement circuit both for CM and DM impedances is provided as the first step. Then, a fine calibration process is explained and validated with passive components. Eventually, the proposed method is exploited for experimental evaluation of the CM and DM noise impedances of an automotive SMPS in a test set-up for conducted emission verification.
\end{abstract}

\section{INTRODUCTION}

The Original Equipment Manufacturers' (OEMs) push for vehicle electrification and autonomous driving fixes new challenges regarding Electromagnetic Compatibility (EMC) in automotive industry. The stringent requirements set by the CISPR 25 standard and by the OEMs themselves often make necessary the use of one or more EMI filters both to limit the conducted emissions of the vehicle noise sources, such as Switched-Mode Power Supplies (SMPS), and to protect the most sensitive components, such as the Electronic Control Units (ECUs). In order to design an effective EMI filter, it is necessary to have a complete knowledge of the surrounding system, especially in terms of impedances.

The impact of external impedances on the noise path and thus on the performance of both passive and active filters in a standard Conducted Emission (CE) test set-up has been studied and analyzed from the theoretical point of view in the literature ( [1], [2]). A schematic representation of the noise path for the Common Mode (CM) and Differential Mode (DM) noise is given in Fig. 1.

The impedance investigation takes more and more importance in active filtering, where the filter might run to instability and act as a noise amplifier if the external impedances are not suitable for the chosen topology, [3]-[6].

Some assumptions are usually considered as a starting point in the EMI filters design:

This work is supported by Schaffner Group, Switzerland. See https://www.schaffner.com/.

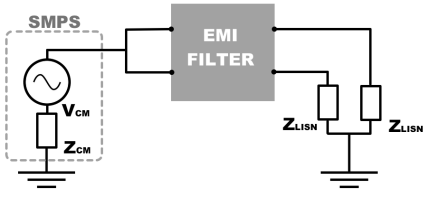

(a) Common Mode

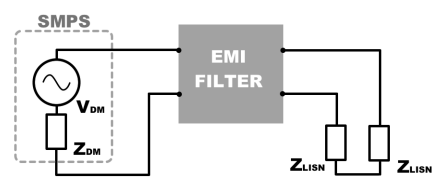

(b) Differential Mode
Fig. 1: Noise circuits.

- The CM impedance is usually small in magnitude on Line Impedance Stabilization Network (LISN) side $(25 \Omega)$ and large on the SMPS side (several $\mathrm{k} \Omega$ );

- The DM impedance is usually small in magnitude on the SMPS side $(1 \Omega-10 \Omega)$ and large on the LISN side (100 $\Omega$ ).

However, these assumptions can only give a rough idea of the behavior of the filter in the real system and they do not give any information about the impedance phase, which is of paramount importance. Furthermore, the LISN behavior is far from being a constant purely resistive $50 \Omega$ component. Namely, the frequency response of the LISN impedance shows an inductive behavior yet for frequencies up to $5 \mathrm{MHz}$, leading to impedance values significantly smaller than the theoretical (resistive) $50 \Omega$ load usually assumed in filter design.

On the other hand, the measurement of the equivalent impedance of a switching device is not immediate and a few methods have been proposed in the literature such as the insertion loss method suggested in [7], and the two probes approach, proposed in [8]-[11].

The former starting from the aforementioned assumptions, allows the derivation of the noise impedance magnitude from noise measurements and then the phase can be retrieved by means of the Hilbert transform. It is a time consuming method which requires many practical measurements and setup changes and can lead to a large error. The latter provides precise results by injecting a current into the system and by measuring with a Vector Network Analyzer (VNA) the $\mathrm{S}$-parameters, but has low accuracy for frequencies below $300 \mathrm{kHz}$.

In this paper, a novel direct approach to the measurement of the complex impedance of a SMPS under loading con- 


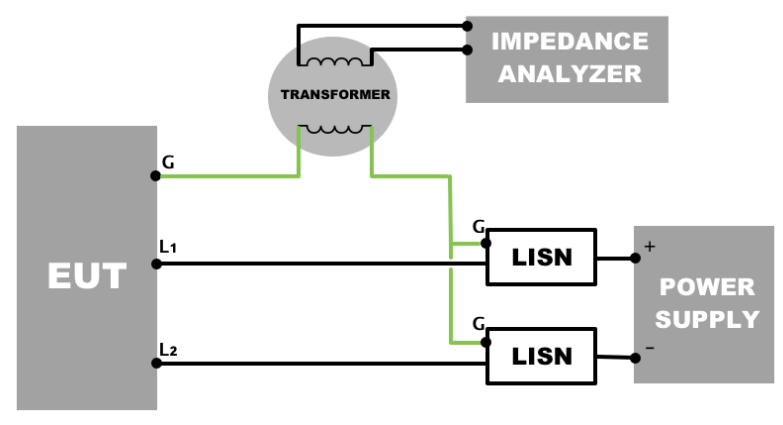

(a) Common Mode

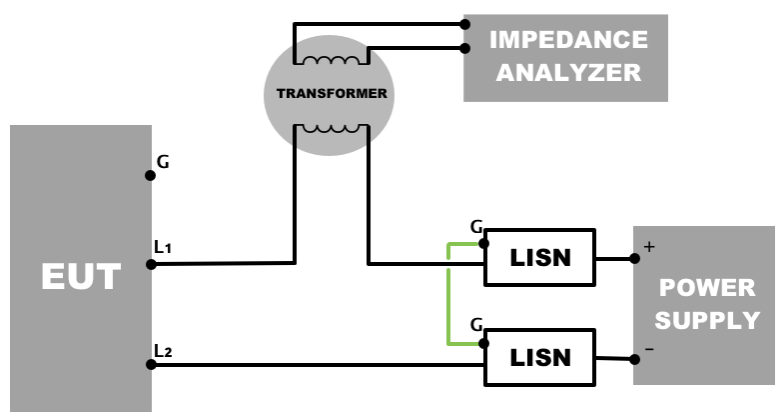

(b) Differential Mode

Fig. 2: Measurement circuits.

ditions is proposed. The method aims at providing accurate measurement of modal impedances both in magnitude and phase by connecting an Impedance Analyzer (IA) directly to the powered system through a transformer. The theoretical study of the measurement circuit for CM and DM impedance characterization is proposed in section II, including a look into the IA measurement mechanism and the most important parameters of the injection transformer. Section III faces the calibration process and the validation of the method with passive components ( $\mathrm{R}, \mathrm{L}$ and $\mathrm{C}$ ). The practical limitations are also discussed. Eventually, in section IV, a real automotive test set-up is built, and the CM and DM noise impedances of a DC/DC converter are measured under different working conditions (i.e., converter powered on and off).

\section{Theoretical Support of the Proposed Method}

With the method proposed in this paper, the impedance values are obtained directly from an IA exploiting its autobalancing bridge architecture, which has a high accuracy over a wide impedance range ( $25 \mathrm{~m} \Omega-40 \mathrm{M} \Omega)$ and it results to be suitable for complex impedance measurements up to $120 \mathrm{MHz}$ [12]-[14].

The electrical schematics in Fig. 2 show the set-up required for the impedance measurement of a generic DUT in the CM and DM configuration. A transformer is connected directly to the output port of the IA, so that the signal generated by the IA oscillator is injected into the system. Referring to Fig. 2, the transformer shall be connected to one of the two power lines to measure the DM noise impedance, while it shall be

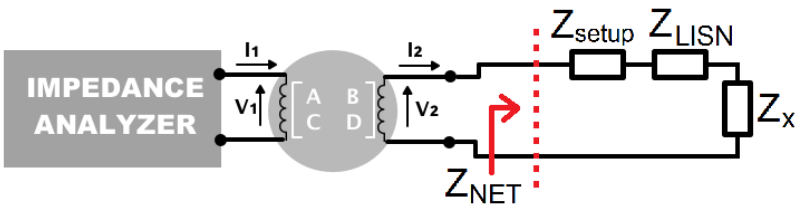

Fig. 3: Transmission matrix representation of the injection transformer.

connected to a dedicated ground wire in order to measure the CM noise impedance. It results more practical to use a dedicated ground wire for the injection since it allows the use of the same transformer both for DM and CM. However, if the grounding is not accessible it is also possible to use a three-winding transformer where both the power lines are wound around the core acting as the transformer secondary side. The injection transformer provides the galvanic insulation required to protect the IA from the DC functional currents and voltages of the Device Under Test (DUT), but it is also the bottleneck of the measurement system and must be chosen carefully. The frequency bandwidth and the saturation current are the two most important parameters to consider and they will be discussed in the next section.

According to [14] and [15], the transformer connected to the output ports of the IA is considered as an additional IA fixture and it can be treated as an unknown 4-terminal network, as shown in Fig. 3, and its transmission matrix results to be:

$$
\left(\begin{array}{l}
V_{1} \\
I_{1}
\end{array}\right)=\left(\begin{array}{ll}
A & B \\
C & D
\end{array}\right)\left(\begin{array}{l}
V_{2} \\
I_{2}
\end{array}\right)
$$

where $V_{1}$ and $I_{1}$ are the output voltage and current of the IA and $V_{2}$ and $I_{2}$ are the actual voltage and current injected into the circuit to be measured. An unknown network impedance measured by the IA is given by the ratio of $V_{1}$ and $I_{1}$ :

$$
Z_{N E T m}=\frac{V_{1}}{I_{1}}=\frac{A V_{2}+B I_{2}}{C V_{2}+D I_{2}}
$$

$Z_{N E T m}$ is the measured value of the impedance, that is different from the actual value. The latter, named $Z_{N E T}$, is the one measured after the transformer and it is given by the ratio of $V_{2}$ and $I_{2}$ :

$$
Z_{N E T}=\frac{V_{2}}{I_{2}}
$$

The relationship between $Z_{N E T}$ and $Z_{N E T m}$ can be derived combining (2) and (3):

$$
Z_{N E T m}=\frac{I_{2}\left(A \frac{V_{2}}{I_{2}}+B\right)}{I_{2}\left(C \frac{V_{2}}{I_{2}}+D\right)}=\frac{A Z_{N E T}+B}{C Z_{N E T}+D}
$$

And finally $Z_{N E T}$ results to be:

$$
Z_{N E T}=\frac{B-D Z_{N E T m}}{C Z_{N E T m}-A}
$$


which is a function of the measured quantity $Z_{N E T m}$ and the transmission matrix of the transformer. From (1), parameters $A, B, C$ and $D$ can be defined as:

$$
\begin{array}{ll}
A=\left.\frac{V_{1}}{V_{2}}\right|_{I_{2}=0} \quad B=\left.\frac{V_{1}}{I_{2}}\right|_{V_{2}=0} \\
C=\left.\frac{I_{1}}{V_{2}}\right|_{I_{2}=0} \quad D=\left.\frac{I_{1}}{I_{2}}\right|_{V_{2}=0}
\end{array}
$$

It follows that an open circuit and a short circuit measurement are required to find the relationship between these parameters. By substituting these current and voltage values in (2), one gets:

$$
\begin{array}{lll}
Z_{o}=\frac{A}{C} & \Rightarrow & C=\frac{A}{Z_{o}} \\
Z_{s}=\frac{B}{D} & \Rightarrow & B=D Z_{s}
\end{array}
$$

where $Z_{o}$ is the open-circuit measured impedance, associated with the magnetizing branch of the injection transformer, whereas $Z_{s}$, i.e., the short-circuit measured impedance, accounts for the leakage inductance and copper losses.

By substituting (7) and (8) in (5) one gets:

$$
Z_{N E T}=\frac{D Z_{s}-D Z_{N E T m}}{\frac{A}{Z_{o}} Z_{N E T m}-A}=\frac{D\left(Z_{s}-Z_{N E T m}\right)}{A\left(Z_{N E T m}-1\right)} Z_{o}
$$

Eventually, to find the relationship between $A$ and $D$, it can be considered that for a symmetrical network, e.g., the transformer used in this work which exhibits the same input impedance at both ports, parameters $A$ and $D$ are equal [14]. The final result is that the network impedance is:

$$
Z_{N E T}=\frac{Z_{s}-Z_{N E T m}}{Z_{N E T m}-Z_{o}} Z_{o}
$$

Therefore, the actual complex unknown impedance $Z_{N E T}$ is derived after recording three direct measurements with the IA, that is: $Z_{o}, Z_{s}$, and $Z_{N E T m}$.

Exploiting the aforementioned procedure it is possible to calculate an unknown impedance $Z_{x}$ in a complex system, e.g., the EUT impedance shown in Fig. 2 and Fig. 3. Considering a standard CE test set-up, it includes a LISN $\left(Z_{L I S N}\right)$, the cables impedance $\left(Z_{\text {setup }}\right)$ and the impedance to be measured $\left(Z_{x}\right)$, which can be either a passive component or a SMPS. By shorting the unknown impedance $Z_{x}$ terminals, the LISN impedances and the impedance introduced by the cables can be recorded:

$$
Z_{N E T_{1}}=Z_{L I S N}+Z_{\text {setup }}
$$

On the other hand, when considering the full system, with $Z_{x}$ connected, the measured impedance is:

$$
Z_{N E T_{2}}=Z_{L I S N}+Z_{\text {setup }}+Z_{x}
$$

Eventually, since the impedances $Z_{N E T_{1}}$ and $Z_{N E T_{2}}$ are in series, the unknown impedance $Z_{x}$ can be calculated as:

$$
Z_{x}=Z_{N E T_{2}}-Z_{N E T_{1}}
$$

\section{A. Theoretical and Practical Limits}

To guarantee accuracy, some considerations must be taken into account. The internal architecture of the IA limits the highest applicable frequency, which in this case is $120 \mathrm{MHz}$. The precision of the measurements depends also on the level of voltage (or current) generated by the IA oscillator, which, according to [14], should be a hundred times (40 dB) larger than the noise voltage (or current) of the system under investigation. This consideration holds only for active systems, e.g., when measuring the impedance of a powered SMPS is the target.

Further limits are set by the injection transformer. First, its frequency behavior and its bandwidth define the frequency band in which measurements are reliable. The other parameter, which becomes important when measuring the DM noise impedance, is the saturation current. For high-current applications, a different material and/or gapped core is necessary. In the system under investigation, which has a high supply voltage $(\sim 450 \mathrm{~V})$ and relatively small current $(\sim 1 A)$, a nanocrystalline magnetic core with three windings is exploited, in order to withstand the DC functional current without saturation, yet providing a wide bandwidth $(10 \mathrm{kHz}-10 \mathrm{MHz})$.

\section{Calibration Procedure and Validation}

A principle drawing of the experimental set-up used for validation is shown in Fig. 3. As explained in Section II, the set-up adopted for DM and CM measurement exploits a slightly different connection of the transformer, thus the validation is repeated for both configurations. In the validation process, three different passive components have been chosen as unknown impedances $Z_{x}$ : a resistor $R_{1}=1 k \Omega$, a capacitor $C_{1}=0.1 \mu \mathrm{F}$, and an inductor $L_{1}=88 \mu H$.

After the machine calibration and the set-up freeze, the same three passive components have been measured by using the same IA with its standard fixture for impedance measurement in the frequency interval from $20 \mathrm{kHz}$ up to $30 \mathrm{MHz}$. These reference quantities $\left(Z_{r e f}\right)$ are compared with those obtained by the proposed method $\left(Z_{I N D}\right)$ in Fig. 4 and Fig. 5 for the $\mathrm{DM}$ and $\mathrm{CM}$ set-up, respectively.

Below $20 \mathrm{MHz}$, the comparison shows an excellent agreement for all experimental points, which fall within a $\pm 5 \%$ interval around the reference values. The small deviations observed around resonance peaks can be interpreted as an effect of small differences in the length of the component leads, giving rise to a shift in the resonance frequency. For frequencies above $20 \mathrm{MHz}$, the observed deviations are to be ascribed to performance degradation of the transformer.

Eventually, the phase error occurring above $10 \mathrm{MHz}$ in the DM measurements seems to be a systematic error, which can be compensated in a more stable test set-up.

\section{Measurements Results}

The test set-up built for the experiments, shown in Fig. 6, is a Conducted Emission test bench, with two automotive certified LISNs (R\&S ESH3-Z6) and an automotive DC/DC converter with ratings: $V_{I N_{\min }}=220 \mathrm{~V}, V_{I N_{\max }}=450 \mathrm{~V}$, 


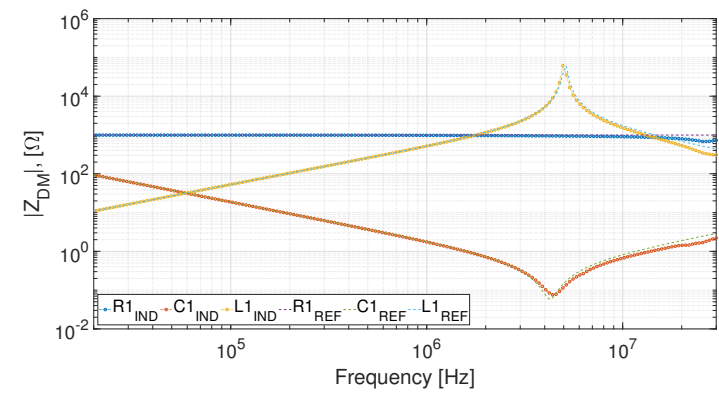

(a) Magnitude.

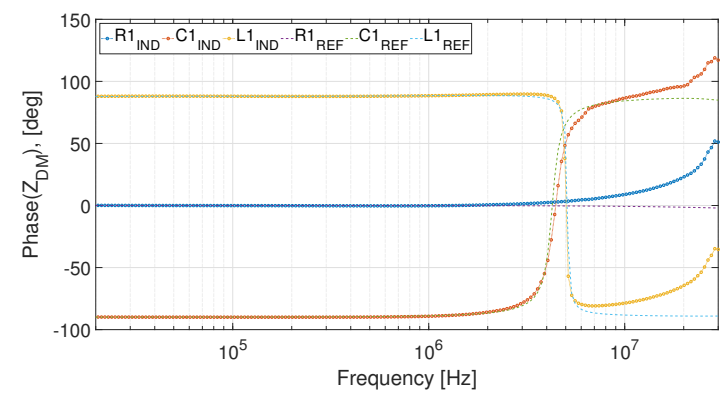

(b) Phase.

Fig. 4: Differential Mode validation with passive components.

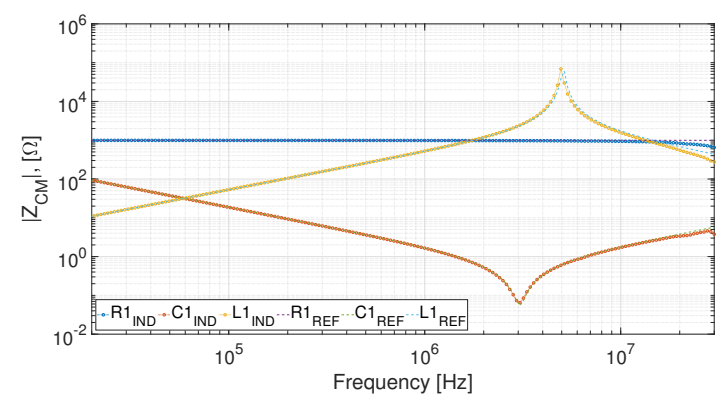

(a) Magnitude.

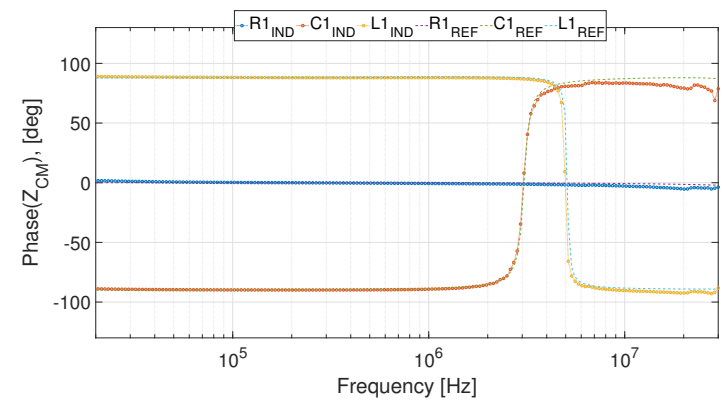

(b) Phase.

Fig. 5: Common Mode validation with passive components.

$V_{O U T}=14 \mathrm{~V}$ and $P_{n}=2 \mathrm{~kW}$. The switching frequency is 80 $\mathrm{kHz}$ and it is a full bridge with isolated low-voltage side. The IA used for the tests is the Keysight E4990A with frequency bandwidth up to $120 \mathrm{MHz}$. The DC/DC converter impedance has been recorded both for DM and CM in different working conditions (e.g., supply voltage, loading, grounding etc.). In

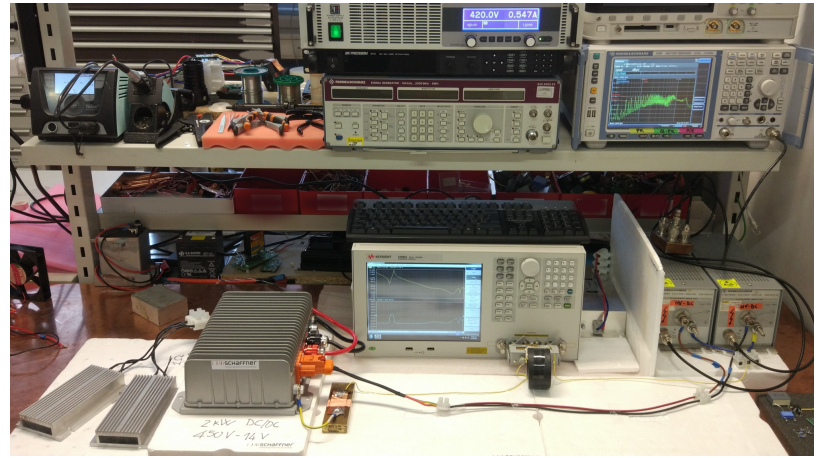

Fig. 6: Test setup used for experimental measurements.

all the following, results obtained with the converter switched on and off will be compared.

\section{A. Differential Mode Experimental Results}

When looking at the DM noise impedance, it is important to understand in what configuration we have access to the actual DM impedance. From the analysis of the electrical circuit in Fig. 2, it results that the actual DM impedance shall be measured without connecting the converter to ground.

Fig. 7 shows the measured impedance when the DC/DC converter is on and off. When it is off, i.e. the blue trace, the impedance corresponds to the one of the input X-capacitors of the SMPS (-20 dB per decade and phase $-90 \mathrm{deg}$ ). When switched on, the SMPS impedance follows the trend of the DM impedances measured in other works found in literature, i.e. $0.1 \Omega-1 \Omega$ between $150 \mathrm{kHz}$ and $1 \mathrm{MHz}$ and then it increases up to hundreds of Ohms. For frequencies above $15-20 \mathrm{MHz}$ the two DM impedances are almost coincident, since their frequency response is dominated by parasitics.

\section{B. Common Mode Experimental Results}

The common mode noise impedance is mainly due to the parasitic capacitance between the switching valves and the heat sink, that is connected to ground [16]. Such a capacitance depends on the physical construction of the SMPS. Hence, it can be observed also when the DC/DC converter is not powered.

This is confirmed in Fig. 8, where the comparison between the CM impedances measured with the converter switched on (red trace) and off (blue trace) is shown. As expected, they are similar and only small differences in magnitude and phase appear between $1 \mathrm{MHz}$ and $10 \mathrm{MHz}$. It is worth to notice that the measurements are taken at $V_{i n}=450 \mathrm{~V}$, that is the maximum operating voltage of the SMPS and it corresponds to the maximum amplitude of the common mode noise. The peaks visible at exactly $80 \mathrm{kHz}$, i.e. the switching frequency of the SMPS, indicates that the CM noise at that frequency is comparable (or even higher) to the measuring signal amplitude injected by the IA and this disturbs the readings. Although not reported here for brevity, this specific feature can be noted only at the maximum operating voltage. If the voltage is decreased, 


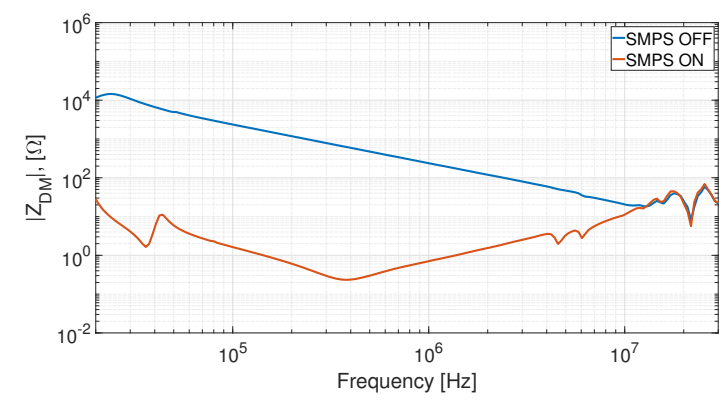

(a) Magnitude.

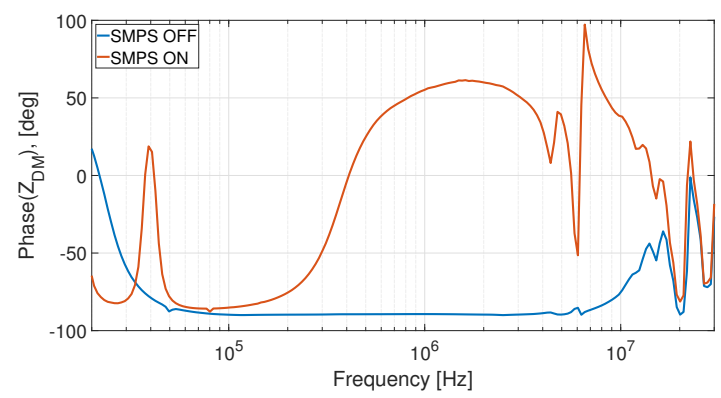

(b) Phase.

Fig. 7: Differential Mode noise impedance: SMPS OFF vs SMPS ON.

for example to $V_{i n}=400 \mathrm{~V}$, the level of the common mode noise decreases as well and thus the disturbance disappears.

\section{CONCLuSion}

In this paper, a novel measurement method to characterize the complex impedance of a SMPS has been presented. This method guarantees high accuracy and allows the measurement of impedance magnitude and phase connecting an Impedance Analyzer directly to a powered system through a transformer.

The technique has been validated with passive components and showed optimal results up to $20 \mathrm{MHz}$, depending on the bandwidth of the transformer in use.

Eventually, the proposed method was exploited to evaluate the CM and DM noise impedances of a DC/DC converter for automotive powertrain application in a test setup for conducted emission verification. The specific results reported in this paper allow appreciating that the power on/off status of the converter significantly influences the frequency response of the DM noise impedance up to $15-20 \mathrm{MHz}$, whereas the $\mathrm{CM}$ noise impedance is almost unaffected.

\section{REFERENCES}

[1] F. Luo, D. Boroyevich, P. Mattavelli, and H. Bishnoi, "Emi filter design considering in-circuit impedance mismatching," in 2012 IEEE Energy Conversion Congress and Exposition (ECCE), Sept 2012, pp. 46134618.

[2] Y. . Son and S.-K. Sul, "Generalization of active filters for emi reduction and harmonics compensation," IEEE Transactions on Industry Applications, vol. 42, no. 2, pp. 545-551, March 2006.

[3] M. L. Heldwein, H. Ertl, J. Biela, and J. W. Kolar, "Implementation of a transformerless common-mode active filter for offline converter systems," IEEE Transactions on Industrial Electronics, vol. 57, no. 5, pp. 1772-1786, May 2010.

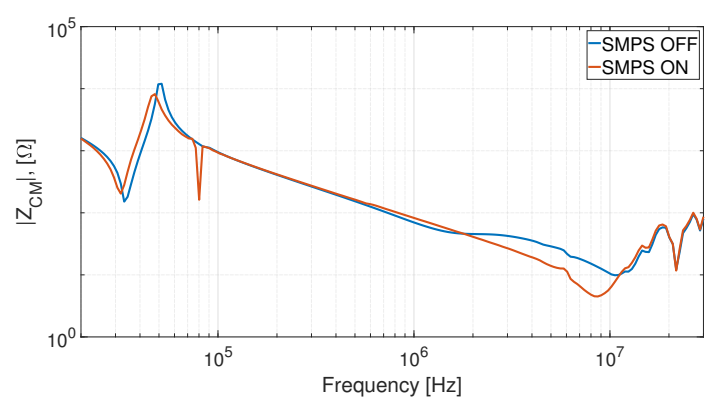

(a) Magnitude.

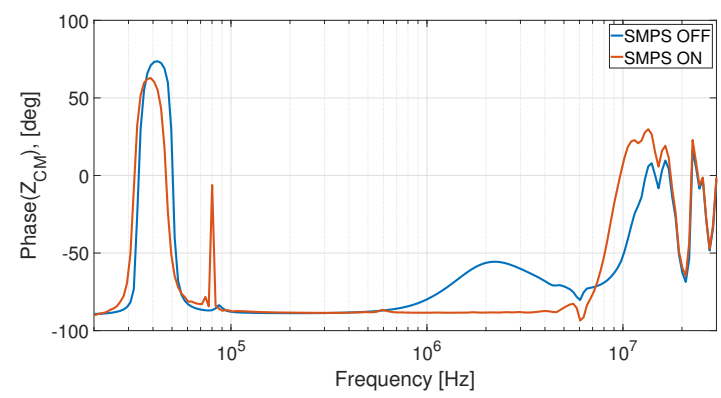

(b) Phase.

Fig. 8: Common Mode noise impedance: SMPS OFF vs SMPS ON.

[4] A. Amaducci, "Design of a wide bandwidth active filter for common mode emi suppression in automotive systems," in 2017 IEEE International Symposium on Electromagnetic Compatibility Signal/Power Integrity (EMCSI), Aug 2017, pp. 612-618.

[5] R. Goswami and S. Wang, "Modeling and stability analysis of active differential-mode emi filters for ac/dc power converters," IEEE Transactions on Power Electronics, vol. 33, no. 12, pp. 10277-10291, Dec 2018.

[6] K. Li, K. See, and F. Fan, "Study of emi filter performance without lisn based on noise impedances," in 2017 Asia-Pacific International Symposium on Electromagnetic Compatibility (APEMC), June 2017, pp. 115-117.

[7] D. Zhang, D. Y. Chen, M. J. Nave, and D. Sable, "Measurement of noise source impedance of off-line converters," IEEE Transactions on Power Electronics, vol. 15, no. 5, pp. 820-825, Sept 2000.

[8] K. Y. See and J. Deng, "Measurement of noise source impedance of smps using a two probes approach," IEEE Transactions on Power Electronics, vol. 19, no. 3, pp. 862-868, May 2004.

[9] V. Tarateeraseth, B. Hu, K. Y. See, and F. G. Canavero, "Accurate extraction of noise source impedance of an smps under operating conditions," IEEE Transactions on Power Electronics, vol. 25, no. 1, pp. 111-117, Jan 2010.

[10] V. Tarateeraseth, "Emi filter design: Part ii: Measurement of noise source impedances," IEEE Electromagnetic Compatibility Magazine, vol. 1, no. 1, pp. 42-49, First 2012.

[11] X. Shang, D. Su, H. Xu, and Z. Peng, "A noise source impedance extraction method for operating smps using modified lisn and simplified calibration procedure," IEEE Transactions on Power Electronics, vol. 32, no. 6, pp. 4132-4139, June 2017.

[12] M. Horibe, "Performance comparisons between impedance analyzers and vector network analyzers for impedance measurement below 100 mhz frequency," in 2017 89th ARFTG Microwave Measurement Conference (ARFTG), June 2017, pp. 1-4.

[13] KeysightTechnologies, Performing Impedance Analysis with the E5061B ENA Vector Network Analyzer - Application Note, 2018. [Online]. Available: http://literature.cdn.keysight.com/litweb/pdf/5991-0213EN.pdf

[14] AgilentTechnologies, Impedance Measurement Handbook, 2013. [Online]. Available: http://www.oh3ac.fi/Impedance_Handbook.pdf 
[15] KeysightTechnologies, Impedance Measurement Handbook, 2016. [Online]. Available: https://literature.cdn.keysight.com/litweb/pdf/ 5950-3000.pdf

[16] M. Maeda, T. Matsushima, T. Hisakado, and O. Wada, "Impedance balance control for reduction of common mode noise in full bridge converter," in 2015 International Conference on Electronics Packaging and iMAPS All Asia Conference (ICEP-IAAC), April 2015, pp. 695-699. 